\title{
O uso das redes técnicas para a gestão de resíduos sólidos
}

Os dados públicos de gerenciamento dos resíduos sólidos no território brasileiro demonstram que a expectativa de maior controle das atividades, a partir da publicação da Política Nacional de Resíduos Sólidos, ainda não foi atendida. A ausência de uma cultura de planejamento e, consequentemente, de disponibilidade de recursos, são possíveis causas da inexistência da gestão integrada e compartilhada de resíduos sólidos, determinada pelo referido instrumento legal. Para garantir a visão sistêmica na gestão integral dos resíduos gerados nos territórios, respeitando às diversidades e potencializando o controle social, faz-se necessário o uso de novas abordagens e estratégias para o planejamento e controle das ações. Nesta perspectiva, o presente artigo objetiva discutir as redes técnicas como um instrumento para a viabilização da gestão integrada e compartilhada de resíduos sólidos. A identificação e qualificação dos agentes sociais, dos fixos e fluxos da rede técnica real, e o reconhecimento da rede técnica proposta pelo arcabouço legal em cada território são camadas que, quando sobrepostos, levam à determinação da rede técnica ideal que realça as especificidades do território e cuja gestão se dá por e para os agentes sociais que as mobilizam. Uma vez que cada rede técnica pode ser utilizada separadamente e em diferentes escalas territoriais, seus limites de utilização serão determinados exclusivamente pelos escopos definidos pelos tomadores de decisão.

Palavras-chave: Resíduos sólidos; Redes técnicas; Gestão de resíduos.

\section{The use of technical networks for solid waste management}

Public data on the management of solid waste in Brazilian territory shows that the expectation of greater control of activities, as of the publication of the National Solid Waste Policy, has not yet been met. The absence of a planning culture and, consequently, the availability of resources are possible causes of the lack of integrated and shared solid waste management, determined by said legal instrument. In order to guarantee the systemic vision in the integral management of the residues generated in the territories, respecting the diversities and potentializing the social control, it is necessary the use of new approaches and strategies for the planning and control of the actions. In this perspective, this article aims to discuss technical networks as an instrument for the feasibility of integrated and shared solid waste management. The identification and qualification of the social agents, of the fixes and flows of the real technical network, and the recognition of the technical network proposed by the legal framework in each territory are layers that, when overlapped, lead to the determination of the ideal technical network that highlights the specificities of the territory and whose management is given by and for the social agents that mobilize them. Since each technical network can be used separately and at different territorial scales, its limits of use will be determined exclusively by the scopes defined by the decision makers.

Keywords: Solid wastes; Technical networks; Waste Management.

Topic: Planejamento, Gestão e Políticas Públicas Ambientais

Reviewed anonymously in the process of blind peer.

\section{Fabiana Alves Fiore}

Universidade Estadual Paulista Júlio Mesquita Filho, Brasil http://lattes.cnpq.br/9905539715619645

fabiana.fiore@ict.unesp.br

Emília Wanda Rutkowski

Universidade Estadual de Campinas, Brasil

http://lattes.cnpq.br/6015770954195537

ewrfec@gmail.com
Received: 20/02/2017

Approved: 22/05/2017
Referencing this:

FIORE, F. A.; RUTKOWSKI, E. W.. O uso das redes técnicas para a gestão de resíduos sólidos. Nature and Conservation, v.10, n.1, p.3948, 2017. DOI: http://doi.org/10.6008/SPC2318-2881.2017.001.0004

DOI: 10.6008/SPC2318-2881.2017.001.0004 


\section{INTRODUÇÃO}

O modo de produção industrial projetado por uma economia não circular é produtor de resíduos, seja pela inviabilidade do uso integral da matéria-prima, seja pela inutilidade do subproduto gerado, seja pelo término da vida útil ou da obsolescência do produto, ou seja, pelo desenvolvimento contínuo de novos produtos. O aumento populacional nas cidades aliado a hábitos consumistas é variável de uma equação de desequilíbrio, cujo resultado é um elevado potencial de degradação ambiental decorrente da quantidade de resíduos gerados (FIGUEIREDO, 1992; FIORE et al., 2008).

Em função dos transtornos às atividades e dos riscos à saúde, os resíduos sólidos precisam ser tratados com a maior brevidade possível. Esse manejo demanda ações de gestão e de gerenciamento cujas especificações e custos, em geral, estão associados à composição do material e à atividade de origem. Após 21 anos de debate parlamentar, a Política Nacional de Resíduos Sólidos, regulamentada pela Lei Federal ${ }^{\circ}$ 12.305/2010, define que a gestão dos resíduos sólidos é de competência pública e deve ser realizada por meio de articulações entre poder público, iniciativa privada e demais segmentos da sociedade civil para concepção, implementação e operacionalização eficaz dos sistemas de resíduos sólidos, conforme o seu art. 10. Sua implementação deve ser integrada, compartilhada, consorciada, regionalizada e integral, a partir de:

1) da visão sistêmica, considerando as variáveis ambiental, social, cultural, econômica, tecnológica e de saúde pública;

2) do norteamento das atividades de manejo para que sejam atendidas as metas previamente estabelecidas em função dos diagnósticos;

3) do controle das atividades de manejo; e

4) da revisão periódica dessas atividades.

Com inspiração processual nos sistemas de gestão ambiental, é proposto o uso da metodologia Ciclo de Demin (PDCA, em inglês) - Planejar, Fazer, Verificar e Agir - por determinar ações sistemáticas cíclicas visando à melhoria contínua (DEMING, 1996; FIORE et al., 2012). Apesar do acolhimento pela sociedade brasileira da PNRS e de sua estratégia de capilarização ao atrelar a verba federal ao setor exclusiva para municípios que construíssem seus Planos Municipais de Gestão Integrada de Resíduos Sólidos (PMGIRS), as transformações ao novo cenário proposto não têm acontecido na velocidade preconizada.

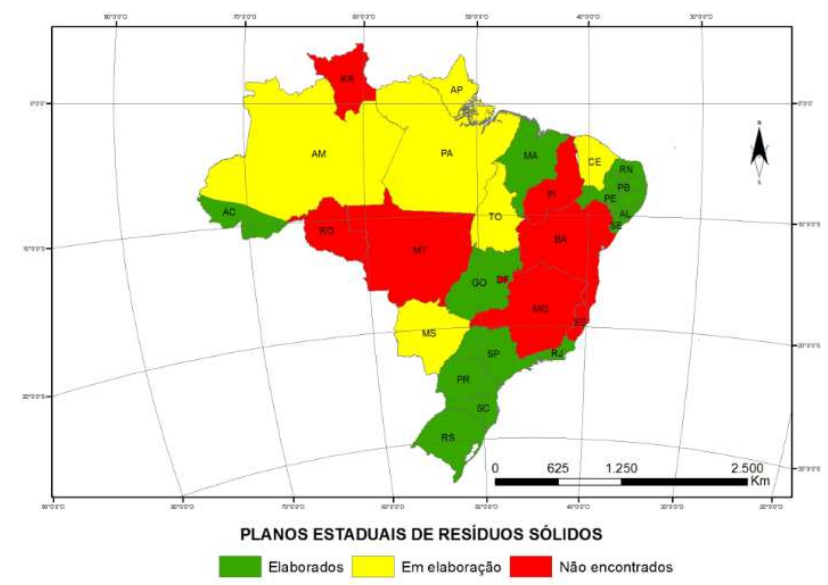

Figura 1: Estágio de elaboração dos Planos Estaduais de Resíduos Sólidos. 
O Plano Nacional de Resíduos Sólidos, discutido nacionalmente durante dois anos, continua como versão preliminar, enquanto vários estados da federação concluíram seus planos estaduais, de acordo com o ilustrado na figura 1, à luz da Política Nacional de Resíduos Sólidos, além de elaborarem programas para disponibilizar apoio técnico e financeiro para a elaboração dos planos municipais. A expectativa era que as práticas municipais de construção do Plano Diretor, em atendimento ao Estatuto da Cidade, regulamentado pela Lei Federal $n^{\circ} 10.257 / 2001$, e de avaliação de processos de licenciamento ambiental fossem utilizadas na construção dos Planos Municipais de Gestão Integrada de Resíduos Sólidos; entretanto, na maioria dos municípios, não ocorreu transversalidade processual entre os gestores públicos locais no planejamento do manejo e tratamento dos resíduos sólidos.

De acordo com a pesquisa de informações básicas municipais, realizada pelo Instituto Brasileiro de Geografia e Estatística em 2013, cerca de 33\% dos municípios brasileiros declararam possuir planos de gestão integrada de resíduos sólidos, em conformidade com a Política Nacional de Resíduos Sólidos. Este reduzido percentual de atendimento a legislação leva à formulação da hipótese desse trabalho: os municípios carecem não apenas de recursos humanos e materiais para a elaboração de seus planos, mas também de instrumentos que viabilizem a efetividade da gestão. Nesta perspectiva, o objetivo do presente artigo é discutir redes técnicas como um instrumento para a viabilização da gestão integrada e compartilhada de resíduos sólidos, em consonância com os princípios, diretrizes e objetivos da Política Nacional de Resíduos Sólidos.

\section{METODOLOGIA}

Este trabalho é fruto de uma pesquisa exploratória onde aplicou-se o método qualitativo que, segundo Leite (2008), permite fazer classificações comparativas e analisar fenômenos considerando-se o contexto. O método enseja a compreensão interpretativa da ação dos agentes sociais no mundo, com profundidade tal que possibilite retratar a complexidade das relações, suas singularidades e contradições (MINAYO, 2005; BAUER et al., 2002). A ordenação dos fenômenos pelo desenho do tipo ideal, preconizada por Minayo (2005), foi à forma do método qualitativo aplicada.

A investigação histórica, com recorte espacial no território brasileiro, foi realizada por meio de pesquisa documental de instrumentos legais e de planos e permitiu o conhecimento das ações de manejo dos resíduos sólidos no território brasileiro (MINAYO, 2005; GODOY, 1995). Os instrumentos legais vigentes sobre o tema foram tomados como discursos do sujeito coletivo, uma vez que figuram aqui como principais acordos sociais possíveis na diversidade brasileira (BAUER et al., 2002).

\section{As redes técnicas de resíduos sólidos}

Utilizadas desde a Antiguidade, as redes permitem ver a trajetória de conexão entre pontos e dão mobilidade aos fluxos. Sua conceituação e teorização surgiram no século XII, mas o desenvolvimento de sistemas que remetem ao sentido de redes é anterior à Era Cristã. Aplicada inicialmente à ecologia para 
explicar as relações entre cada um dos elementos (nós) de um sistema, a teoria de redes criou concepções e clima intelectual que ensejaram avanços científicos significativos (CAPRA, 1996; MUSSO, 2005; COSTA, 2008).

Sua capacidade de responder a diferentes problemas em diversos domínios fez com que o conceito fosse aplicado frequentemente em muitas áreas. No século XXI, as redes foram percebidas como transladadas na sociedade, nas relações sociais e nas relações tecnológicas e virtuais, sendo empregada em vários campos do conhecimento (WASSERMAN et al., 1999; SPUDEIT, 2010).

As redes territoriais, aqui tratadas, foram objetivadas como matriz técnica, que modificam a relação espaço-tempo (MUSSO, 2004). Em suas dimensões de matéria e essência, as redes preservam a característica de conectividade podendo então ser entendidas como uma forma particular de organização e como um instrumento que viabiliza circular e comunicar. A instabilidade das conexões que altera continuamente a estrutura das redes, em função da interação entre os elementos que as compõem, é o que permite a sua adaptação às condições do ambiente e às dinâmicas dos territórios (DIAS, 1995; SANTOS, 1994; SILVEIRA, 2003).

Consideradas as características das redes técnicas e dos elementos que compõem os sistemas de gestão de resíduos sólidos, pode-se afirmar que as redes técnicas de resíduos não foram ainda reconhecidas. O fluxo de resíduos que ocorre entre os fixos assim como as informações que circulam entre os agentes sociais, tanto os responsáveis pela gestão do sistema como seus usuários, são os elementos que compõem e mobilizam essa rede técnica. Sob a ótica da gestão, a rede técnica de resíduos sólidos pode ser melhor descrita como uma tipologia em tríade onde a realidade, o regramento jurídico e o cenário idealizado coexistem e interagem em distâncias passíveis de serem mensuradas ao longo do tempo. Estas três camadas da rede de resíduos sólidos foram denominadas: rede técnica real, legal e idealizada.

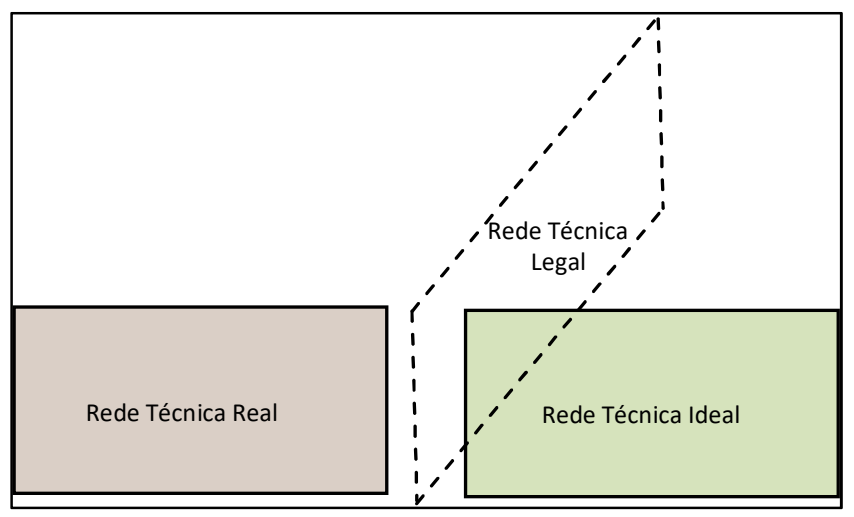

Figura 2 - Interconectividade entre os tipos de rede técnica.

\section{Rede Técnica Real}

A rede técnica real, "produto e suporte da atividade social" (SILVEIRA, 2003) é fruto das ações de manejo dos resíduos nos territórios e espelham o contexto político, social e econômico do lugar. É composta por agentes sociais que demandam, utilizam e operam os sistemas por meio dos fixos ${ }^{1}$, bem como matéria, valores e informações que se põem em circulação.

${ }^{1}$ Objetos técnicos (fixos no espaço) onde são gerados, manejados e realizada a gestão dos resíduos sólidos. 
A população é um dos principais agentes sociais geradores de resíduos sólidos, no qual as especificidades dos resíduos gerados são decorrentes das atividades realizadas, do perfil socioeconômico do indivíduo e do seu meio de inserção. No Brasil, de modo geral, os geradores segregam, acondicionam e armazenam temporariamente os resíduos sólidos nos próprios fixos onde foram gerados. Com exceção do uso domiciliar e de espaços públicos, nos outros fixos, cabe ao agente gerador a responsabilidade por todas as demais atividades de manejo dos resíduos sólidos. Nesse caso, ele é também um mobilizador da rede técnica.

Os agentes sociais que operam as atividades de manejo desenvolvem suas atividades em partes específicas da rede. Esses agentes são os coletores formais e informais, os responsáveis pela limpeza pública (varrição, poda, capina etc.) e privada, os beneficiadores dos materiais reversos e os operacionalizadores da disposição final. Os agentes sociais gestores de diferentes instâncias de poder determinam os componentes fixos e logísticos da rede técnica; têm assento em repartições diversas, uma vez que as ações sobre os resíduos sólidos intervêm diretamente nas condições de saúde coletiva e do meio, bem como da infraestrutura urbana.

A rede técnica é composta por um sistema de fixos que se integram para fazer circular matéria, serviços e informação. Os elementos físicos que suportam as atividades relacionadas à geração de resíduos, beneficiamento e disposição são os objetos técnicos fixos da rede técnica de resíduos sólidos. Todas as atividades humanas geram resíduos sólidos com características específicas. Nestes fixos, o gerador dos resíduos determina aqueles que são impróprios para uso. Esta geração pode ter característica difusa em áreas de uso coletivo. Nos fixos de geração, pode haver outras atividades relacionadas aos resíduos, como o beneficiamento e mesmo a disposição. Todavia, considerando que os resíduos sólidos não são o objetivo das ações humanas e que sua destinação in situ pode conflitar com os demais usos do espaço, em geral, no local de geração, eles são apenas segregados, acondicionados e armazenados temporariamente.

O beneficiamento dos resíduos sólidos pode ocorrer em quase todos os fixos da rede, mas existem unidades destinadas especificamente a isso e que, portanto, foram categorizadas neste trabalho como fixos beneficiadores. Esgotadas as possibilidades de minimização, reuso e reciclagem, os rejeitos são dispostos no solo. A disposição pode ser temporária ou permanente, de maneira apropriada (legal) ou inadequada (clandestina). Por entre os fixos da rede técnica, ocorre o fluxo reverso de resíduos e rejeitos.

Os limites desta rede técnica são tão variáveis quanto forem os arranjos sociais, institucionais e administrativos, pois estes definem a distribuição de bens e produtos e o compartilhamento de soluções para beneficiamento e disposição dos rejeitos. O recorte espacial, como município, região metropolitana, regiões consorciadas ou bacia hidrográfica, é um recorte para otimizar as informações existentes. Esses espaços não são meros limites geográficos, mas território, pois são seu uso e sua apropriação pelos agentes sociais que darão conformidade à rede técnica (SANTOS, 2008). Apropriando-se do espaço de uso, os indivíduos configuram as diversidades locais e regionais sociais, ambientais, econômicas e políticas que são determinantes para a estruturação da rede técnica. 
Os fixos e fluxos da rede técnica real não são definidos somente pelas diretrizes da gestão pública de resíduos sólidos. Há atividades sendo realizadas que são pouco reconhecidas pelo sistema, como a ação dos catadores de materiais recicláveis e reutilizáveis e os descartes irregulares. Ao transpor os limites políticoterritoriais contínuos, ela pode determinar significativos impactos em regiões distantes daquelas onde os resíduos foram gerados. Portanto, o reconhecimento da rede técnica real ilumina, sob a ótica dos agentes sociais, as dinâmicas que as propiciam.

\section{Rede Técnica Legal}

O arcabouço legal sobre a temática norteia soluções ambiental e socialmente adequadas, consideradas as condições políticas, econômicas e culturais. Em muitos casos, as soluções previstas em instrumentos legais detalham atividades de gerenciamento e unidades passíveis de servirem a tal fim. Dessa forma, é possível vislumbrar os prováveis caminhos dos resíduos desde o ponto de geração até a disposição final. Pela análise dos instrumentos legais, é possível identificar as possíveis unidades gestoras, geradoras, beneficiadoras e de disposição dos resíduos sólidos que, quando associadas ao território, dão origem aos fixos da rede técnica. Por apresentar soluções adequadas ao gerenciamento dos resíduos, a fixação dessa imagem de rede técnica no território pode determinar a rede técnica ideal.

A imagem de rede técnica ideal construída pelos instrumentos legais - aqui denominada rede técnica legal - é abrangente e indica as alternativas de beneficiamento e disposição dos resíduos sólidos, respeitadas as características territoriais. Além de apresentar os fixos ideais predominantes, a rede técnica legal também orienta o fluxo dos resíduos sólidos visando à minimização do transporte e do impacto ambiental associado. Essa qualificação de fixos e fluxos enseja um processo plenamente cooperativo de gestão. A devida caracterização dos agentes sociais só é possível na rede técnica real, uma vez que ela assume contornos locais. Grande parte das informações e valores que circulam na rede técnica de resíduos sólidos constam nos instrumentos legais e é descrita como objetivos, princípios e diretrizes.

Em geral, os instrumentos legais que versam sobre o tema são elaborados por equipes de profissionais da área que, considerando as condições reais e as alternativas técnicas e tecnológicas de minimização dos impactos, dão as possíveis alternativas de gestão e gerenciamento dos resíduos. Essa elaboração representa os acordos sociais possíveis num dado período. Assim, a rede técnica legal determina condutas e ações e deve ser interpretada como uma rede em evolução, como o é a própria sociedade que a estabelece e adota. Mesmo porque, tanto os resíduos quanto as tecnologias de beneficiamento e disposição sofrem constantes alterações e aí está o maior ganho de se tratarem os requisitos legais que versam sobre o tema sob a perspectiva das redes técnicas: a apropriação da rede técnica legal de fixos e fluxos ideais permite avaliar suas possibilidades de mudança.

A rede técnica legal é a resultante das diretrizes legais aplicáveis ao território analisado. Assim sendo, no Brasil ela é a somatória dos regramentos da esfera federal, estadual e daquelas vigentes no próprio município. Considerada a diversidade de requisitos legais sobre a temática a descrição da rede técnica legal, para cada uma das classes de resíduos sólidos em estudo, permite uma maior especificação dos componentes 
da rede. Dentre as redes técnicas legais determinadas pela legislação brasileira está a de resíduos de serviços de saúde, mostrada na Figura 3, a título de exemplificação.

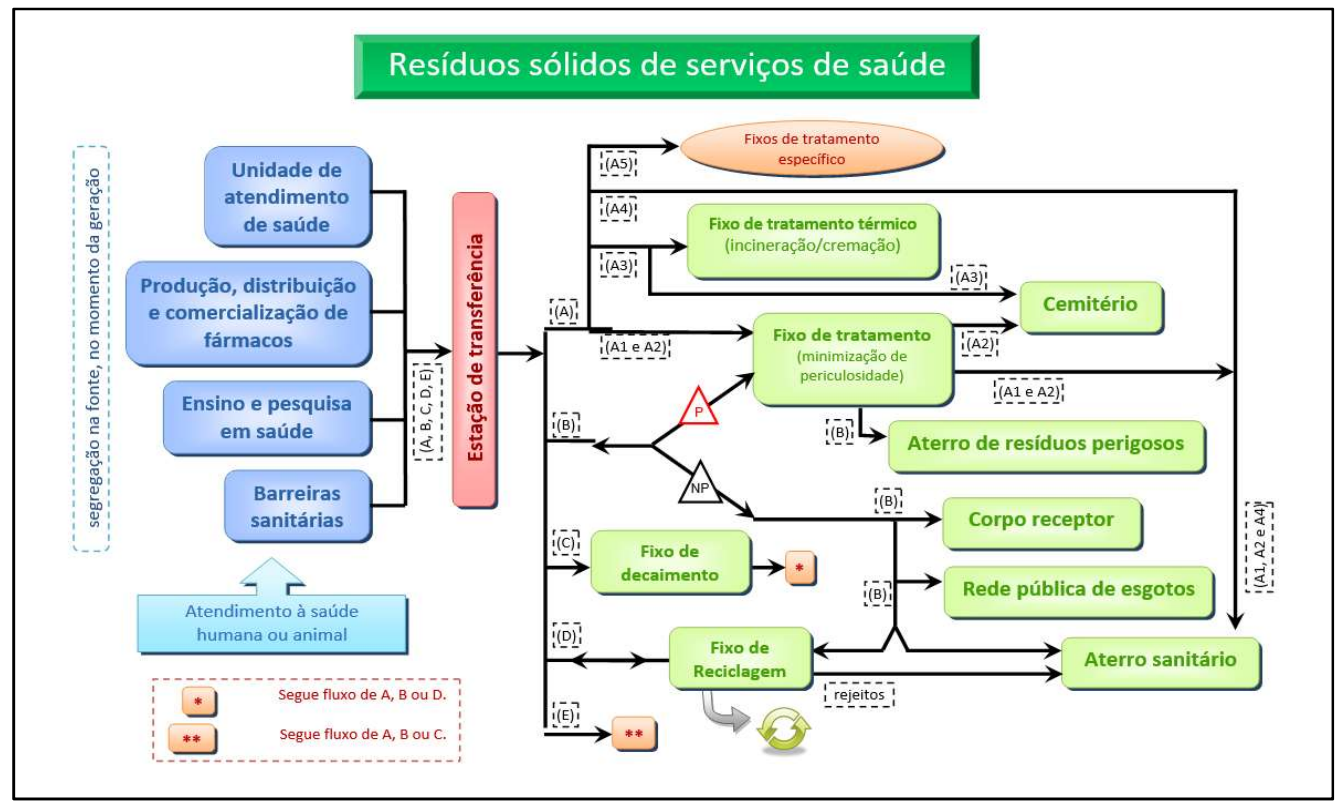

Figura 3: Rede Técnica Legal de Resíduos de Serviços de Saúde.

Fonte: CONAMA, 2005.

\section{Rede Técnica Idealizada}

No contexto da ecologia industrial, uma rede técnica específica de resíduos sólidos não existiria, pois os fixos e fluxos envolvidos no pós-consumo estariam incluídos nas redes de produção, sobrando os rejeitos. No atual estágio de desenvolvimento humano as redes técnicas idealizadas de resíduos sólidos são aquelas em que a geração é a menor possível - considerando-se os pactos sociais vigentes no território -e se minimizam os impactos decorrentes da disposição dos rejeitos. Portanto, o princípio determinante da rede técnica idealizada é minimizar os impactos ambientais e sociais do território onde ela se insere.

Em geral, a legislação aplicável aos resíduos sólidos orienta o gerenciamento das atividades nesse sentido, consideradas as limitações econômicas e tecnológicas. Assim, determina a implantação de fixos adequados para o beneficiamento e a disposição dos resíduos que, quando também são devidamente operados, podem ser considerados suficientes para a rede técnica idealizada.

Fixos ideais de gestão, beneficiamento e disposição, assim como fluxos adequados mínimos, configuram a rede técnica idealizada de resíduos sólidos em períodos determinados. Nessa rede idealizada, circula a menor quantidade possível de resíduos, pois são revertidos para novos processos produtivos e os rejeitos são dispostos adequadamente. O fluxo de informações nesta rede tem papel preponderante. As cadeias produtivas devem se entrelaçar de modo que os resíduos de uma seja matéria prima em outra, base para a economia circular. Os processos produtivos que ainda geram resíduos devem viabilizar sua destinação de modo a evitar o aterramento, estimulando os geradores a uma produção mínima.

A rede técnica idealizada é o algoritmo resultante da sobreposição das redes técnicas real e legal em cada território. Por meio dela se constrói o planejamento e o plano municipal de gestão integrada de resíduos 
sólidos. Por meio da rede técnica idealizada podem-se identificar os conflitos para a implantação de um sistema de gestão territorial de resíduos sólidos que atenda aos princípios de universalização, integralidade, sustentabilidade econômica e qualidade previstos na Política Nacional de Saneamento, regimentada pela Lei Federal 11.445/2007.

\section{As potencialidades da rede técnica de resíduos sólidos}

As redes técnicas estruturam, implementam e operacionalizam sistemas de gestão de resíduos sólidos, que buscam melhoria contínua. Deming (1986) propôs que a melhoria contínua deveria ser avaliada em ciclos por meio de quatro passos - Planejar, Executar, Checar e Agir [PECA ou PDCA, em inglês]. Nesta perspectiva, pode-se inferir que:

Planejar: é a descrição das redes técnicas real e legal e definição de indicadores de desempenho;

Executar: é o momento de implementação de fixos; avaliação dos fluxos de matéria e informações, organização do banco de informações e desenvolvimento de ações de sensibilização voltadas aos agentes sociais;

Checar: é o momento de avaliação da rede técnica, por meio dos indicadores de desempenho determinados no planejamento, para identificação de oportunidades de evolução, criação de novas redes técnicas e determinação de execução de medidas preventivas e corretivas;

Agir: reavaliação do sistema visando à melhoria do desempenho e revisão do sistema de gestão de resíduos.

Apesar de os municípios possuírem responsabilidade de manejo exclusivamente dos resíduos sólidos urbanos, o poder público municipal deve reconhecer as redes técnicas de resíduos como de construção civil e de serviços de saúde para que sua gestão não degrade o ambiente nem ponha em risco a saúde das comunidades e do meio ambiente. Além disso, o controle social preconizado pela Política Nacional de Resíduos Sólidos, deve considerar todo o sistema e seu complexo de redes técnicas com indicadores que, segundo Gallopin (1996), sejam politicamente aceitos no nível adequado para que a avaliação seja capaz de influenciar os tomadores de decisões. A avaliação do sistema por meio dos ciclos de melhoria contínua [Figura 4] permite uma análise crítica contínua do sistema implantado de modo a subsidiar criteriosamente os processos de controle social.

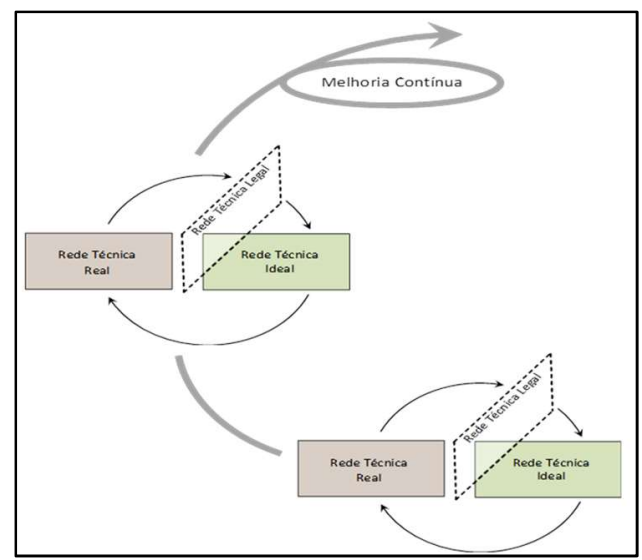

Figura 4: Ciclo de melhoria do Sistema de Gestão de Resíduos Sólidos. 


\section{CONSIDERAÇÕES FINAIS}

A gestão de resíduos sólidos determinada pela Política Nacional de Resíduos Sólidos se fundamenta em princípios e diretrizes de redução de geração, reuso, minimização e reversão dos materiais que não são atendidos pelos modelos vigentes usuais. Nestes, as ações se voltam exclusivamente para a destinação menos impactante e segura, pois os resíduos sólidos são aceitos como inerentes ao desenvolvimento e um direito individual. A gestão sugerida pela Política Nacional de Resíduos Sólidos vai muito além da operacionalização das atividades de manejo: inclui mudança de cultura, hábitos dos cidadãos.

A construção do processo de gestão por meio das redes evidencia as conexões temporais entre os nós do sistema, viabilizando as melhores condições de fluxo permitidas pelas técnicas e tecnologias existentes. Além disso, a gestão se dá por e para os agentes sociais que as mobilizam. As redes técnicas de resíduos sólidos são realidades ainda não plenamente iluminadas, mas sua devida descrição permitirá o controle integral do sistema, a participação e a inclusão social, além de garantir os subsídios para a melhoria contínua. As dimensões da rede técnica de resíduos sólidos, assim como o consumo energético necessário à sua manutenção, podem servir para contextualizar o grau de desenvolvimento local, e seus custos estão associados à sua evolução e eficiência.

A mudança de cenário prevista pela Política Nacional de Resíduos Sólidos e a incapacidade de atendimento ao regramento legal concorrem para que a estratégia redes técnicas seja apropriada pelos gestores públicos e agentes sociais. Uma vez que cada rede técnica pode ser utilizada separadamente e em diferentes escalas territoriais, seus limites de utilização serão determinados exclusivamente pelos escopos definidos pelos tomadores de decisão.

Ressalta-se que o uso da rede técnica real se presta à realização do diagnóstico ambiental necessário à elaboração dos planos de gestão e das avaliações de desempenho ambiental do sistema implantado. A determinação da rede técnica legal estabelece o padrão de qualidade mínimo a ser atingido em cada um dos nós da rede e que a rede técnica idealizada

\section{REFERÊNCIAS}

BAUER, M. W.; GASKELL, G.. Pesquisa qualitativa com texto, imagem e som: um manual prático. Petrópolis: Vozes, 2002.

CAPRA, F.. A teia da vida. São Paulo: Cultrix, 2004.

CONAMA. Conselho Nacional do Meio Ambiente. Resolução n.307 de 5 de julho de 2002.

Estabelece diretrizes, critérios e procedimentos para a gestão dos resíduos da construção civil. Brasília: DOU, 2002.

CONAMA. Conselho Nacional do Meio Ambiente. Resolução n.358 de 29 de abril de 2005. Dispõe sobre o tratamento e a disposição final dos resíduos dos serviços de saúde e dá outras providências. Brasília: DOU, 2005.

COSTA, J. M.. Redes técnicas e uso privado do território na Amazônia: o caso da Estrada de Ferro Carajás da Companhia Vale do Rio Doce (1997-2006). Dissertação (Mestrado em Geografia) - Universidade Federal do Rio Grande do Sul, Porto Alegre, 2008.

DEMING, W. E.. Out of the Crisis. 2 ed. Cambrigde: MIT, 1986.

DIAS, L. C.. Redes: emergência e organização. In: 
CASTRO, I. E.. Geografia: Conceitos e Temas. Rio de Janeiro: Bertrand Brasil, 1995.

FIGUEIREDO, P. J. M.. Os resíduos sólidos e sua significância frente ao impasse ambiental e energético da atualidade. Tese (Doutorado em Planejamento de Sistemas Energéticos) Universidade Estadual de Campinas, Campinas, 1992.

FIORE, F. A.; RUTKOWSKI, E. W.. Gestão e gerenciamento de resíduos sólidos no brasil: os princípios e as diretrizes dos instrumentos legais brasileiros. In: CONGRESSO INTERAMERICANO DE RESÍDUOS SÓLIDOS DA DIRSA/AIDIS, 5. Anais. Lima, 2012.

FIORE, F. A.; RUTKOWSKI, E. W.; MENDONÇA, M. I. G. M.. Gestão de resíduos sólidos de construção civil no município de São José dos Campos/SP. In: CONGRESSO INTERAMERICANO DA AIDIS. Anais. Santiago, 2008.

GALLOPIN, G. C.. Environmental and Sustainability Indicators and the Concept of Situational Indicators: A System Approach. Environmental Modelling \& Assessment, n.1, 1996.

GODOY, A. S.. Introdução à Pesquisa Qualitativa e suas possibilidades. Revista de Administração de Empresas, São Paulo, v.35, n.2, 1995.
LEITE, F. T.. Metodologia científica: métodos e técnicas de pesquisa (monografia, dissertação, teses e livros). Aparecida: Ideias \& Letras, 2008.

MINAYO, M. C. S.. Avaliação por triangulação de métodos: abordagem por problemas sociais. Rio de Janeiro: Fiocruz, 2005.

MUSSO, P.. A filosofia da rede. In: PARENTE, A.. Tramas da rede. Porto Alegre: Sulinas, 2004.

SANTOS, M.. Técnica, espaço, tempo: globalização e meio técnico-científico informacional. São Paulo: Hucitec, 1994.

SANTOS, R. F.. Planejamento ambiental: teoria e prática. São Paulo: Oficina de Textos, 2004.

SILVEIRA, L. L. R.. Redes e território: uma breve contribuição geográfica ao debate sobre a relação sociedade e tecnologia. Revista Bibliográfica de Geografía y Ciencias Sociales: Universidad de Barcelona, v.8, n.451, 2003.

SPUDEIT, D. F. A. O.. O fenômeno das redes de informação: reflexão teórica. Revista ACB, Florianópolis, v.15, 2010.

WASSERMAN, S.; FAUST, K.. Social Network Analysis: Methods and Applications. Cambridge: Cambridge University Press, 1999. 